\title{
Aquaculture production optimization in multi-cage farms subject to commercial and operational constraints
}

\begin{abstract}
Over the past few decades, aquaculture production has grown continually as a result of advances in new production methods to become an alternative to meet the growing global demand for fish within the context of depletion of fisheries resources. In this new context, market competition has increased and the complexity of managing industrial-scale production processes involving biological systems is still a growing problem in aquaculture. This has led, in many cases, to a lack of management capacity. This paper presents a methodology that integrates a multi-criteria model and a Particle Swarm Optimization (PSO) technique with the aim of finding a production strategy that optimizes the value of multiple objectives at a fish farm with multiple batches, cages, feeding alternatives and products. The approach first considers not only the effect of biological performance on economic profitability, but also the effect on environmental sustainability and product quality aspects. The model developed in this paper also constitutes a novelty, as it represents a first attempt to address the optimization of all the operational activities at a farm via artificial intelligence techniques. It includes the consideration of new operational and commercial constraints, such us the maximum volume of fish harvested per week, based on labour and marketing constraints, or the minimum volume of fish harvested on specific dates necessary to comply with commercial agreements. The results demonstrate the utility of this novel approach to decision-making optimization in aquaculture both when establishing overall strategic planning and for integrating new production methods.
\end{abstract}

\section{Keywords}

Aquaculture management, Biosystems, Multi-criteria modelling, decision-making processes, Particle Swarm Optimization.

\section{Introduction}

Over the last few decades, major developments in the new information and communication technologies (ICT) has allowed producers to greatly improve their management capacity in the vast majority of productive sectors, as well as in primary industries. During this time, aquaculture production has become a fast-growing food production industry as a result of advances in new intensive production methods. However, specific techniques to support operational management in this industry have not been developed to the expected extent in a new and expanding industry that is highly dependent on biological and environmental factors. Despite the fact that interest in bio-economic models that simulate the cultivation process has increased lately (Llorente and Luna, 2016; Granada et al., 2018), aquaculture management has yet to see sufficient development of techniques to better understand and optimize decision-making processes. This problem has become even more serious in recent years for the reason that the simulation models and optimization techniques that have traditionally been applied are no longer adequate to efficiently handle the large volumes of data and increasing number of factors involved in this activity.

In terms of the complexity of aquaculture production processes, major research efforts have been made over the past 30 years focused on understanding biological aspects or looking for empirical relationships in the fattening process. As a result, a number of parameters have been identified as the main aspects to model fish growth with the aim of increasing profitability, such as water temperature and feed ration (Ido Seginer, 2016). However, most studies do not allow managers to go beyond default bioeconomic models in order to consider the new objectives increasingly 
demanded by stakeholders, such as environmental sustainability and product quality. For this reason, future methods for fish farming need to be more advanced and smarter in the sense that the industry needs to shift from experience-driven to knowledge-driven approaches so as to better optimize production (Føre et al., 2018)

In this respect, multiple-criteria decision-making (MCDM) techniques have already proven effective when integrating various criteria in order to establish rankings of alternatives in many sectors (Ishizaka et al. 2011). Furthermore, they have been successfully applied in many domains where decisions have to be made in the presence of multiple objectives and subjective criteria which usually enter into conflict, as in the case of aquaculture (Tzeng and Huang, 2011). However, several review papers, from Mardle and Pascoe (1999) to Mathisen et al. (2016), have highlighted the few publications on multi-criteria decision-making within this sector compared to other fields. Moreover, in those cases in which this approach has already been applied, it only addresses very specific problems, such as site selection (Dapueto et al. 2015; Shih, 2017).

On the other hand, the process of feeding fish is increasingly carried out in large facilities, with many production units (cages) that are at different stages of their product life cycle. This has improved the possibilities and efficiency of the sector, but at the same time has increased its complexity and market competitiveness. Different management tools and Decision Support Systems (DSS) have addressed this problem, providing expert information in an easy-to-use manner to end users. However, as stated by Cobo et al. (2018), there is a need to consider their application to large farms, with more than one production unit as well as several supply agreements with large retailers that demand a continuous supply of produce throughout the year. In this regard, these methodologies or systems have to be capable of sequencing seeding and harvesting decisions among multiple production units and cultivation cycles, considering different constraints in order to be practically applicable to establishing an optimal strategic plan.

For all the above reasons, the central goal of this paper is to provide aquaculture producers with a model to address their decision-making throughout the entire production process that enables more efficient management of both small and large aquaculture companies. This goal entails modelling the production process to simulate the strategic plan of a company with multiple cages, multiple cycles, multiple feedstuffs and multiple fish products, optimizing it towards multiple objectives. This implies analysing the effects of each decision on the main variables of a farm. However, optimizing the entire production process of a company by synchronizing seeding and harvesting decisions also implies taking into account operational and commercial constraints, i.e. the maximum amount that the company's workers could harvest per day or the maximum selling volume for the company at the market price, making the challenge even tougher.

To this end, a novel methodology has been developed and tested that integrates a multi-criteria model and an Artificial Intelligence (AI) metaheuristic technique called Particle Swarm Optimization (PSO) The methodology starts with the implementation of a biological model as the basis of three submodels, based on the methodology developed by Luna et al. (2019a), with the aim of analysing the effect of the biological performance of a farm on three crucial aspects: its profitability, its effect on the environment, and the quality of its final product. This allows us to formulate an objective function and conduct a process of finding the optimal production strategy based on multiple objectives. Like most real-world optimization processes, this process is very complex and time consuming, so conventional optimization techniques could encounter many difficulties when attempting to address it. To overcome any such problem, this paper also uses PSO, a population-based stochastic optimization technique inspired by the social behaviour of groups of animals. Although PSO has been successfully applied to solving many multi-objective 
problems (Arion de Campos, 2019), there have only been a few applications in aquaculture, such as those by $\mathrm{Yu}$ and Leung $(2005,2009)$ and Cobo et al. $(2015,2018)$. This technique allows the methodology developed here to start out from a series of alternative strategies or candidate solutions and, based on the results estimated by the model, advance in the search for a near optimal solution with a low computational cost.

This paper thus constitutes a novel contribution to the existing state of the art of precision fish farming, both in terms of the understanding and modelling of the different processes involved and the application of AI techniques to the aquaculture decision-making process. The rest of the paper is structured as follows. First, Section 2 explains the methodology we have developed, while Section 3 elucidates the model. The model is then tested in Section 4 for the case of gilthead seabream farming under three scenarios with commercial and operational constraints. To conclude, Section 5 discusses the multi-criteria model and the optimization technique that allow us to achieve these results.

\section{Simulation and optimization methodology}

This section presents the work carried out to develop a new modelling and simulation methodology with the aim of addressing the current problems of aquaculture producers, as explained above.

In this regard, although these methods could be applied to the cultivation of the vast majority of aquaculture species, the present study started by addressing the entire fattening process of gilthead seabream (Sparus aurata) and European seabass (Dicentrarchus labrax). The selection of these species was the result of a comprehensive analysis of the industry, in which the process of breeding these species is relatively recent, but has undergone rapid growth over the last few years. This means that unlike other species such as salmon, the process of cultivating these fish is still at an initial stage and hence faces more problems of profitability and difficulties in reducing production costs, mainly due to the existence of many small companies and the overwhelming influence of external factors (Llorente and Luna, 2013).

One of the promising solutions to this lack of efficiency is the possibility of taking advantage of advances in information technologies to improve management processes. This would make it possible to carry out this process more efficiently at aquaculture facilities with a large number of floating sea cages. Furthermore, a suitable simulation model would also make long-term forward planning possible, which is very important for the reason that each fingerling has to be fattened for about one year to reach the minimum commercial weight. Therefore, the development of methods and systems of this kind would constitute an even greater contribution to the improvement of decision-making process in this context.

Regarding this aim, each cage at the farm will have an individual strategy that consists of several cultivation cycles (batches), with the assumption that a batch cannot be stocked until the previous one has been harvested, synchronized by their respective seeding date ( $\mathrm{Sd}$ ) and harvesting date (Hd). This also implies the selection of the product $(\mathrm{Pt})$ the farmer wishes to sell between seabream and seabass, the initial weight of the fish fingerlings $(\mathrm{Fw})$ and the feeding decision $(\mathrm{F})$. The overall company profits are subsequently estimated from the results for each cage (Fig. 1). Moreover, it is also essential to first test the validity of the entire strategic plan in terms of the farm's operational and commercial capacity, represented as a range in which the maximum volume of harvested fish per week, based on labour and marketing constraints, and the minimum 
volume of fish sold on specific dates, in order to comply with the commercial agreements that the 135 producer has with recurrent buyers, are established.

136 Once the simulation model was developed, a metaheuristic optimization technique was used to address the complex problem of finding a near optimal strategy with an acceptable computational 138 cost.

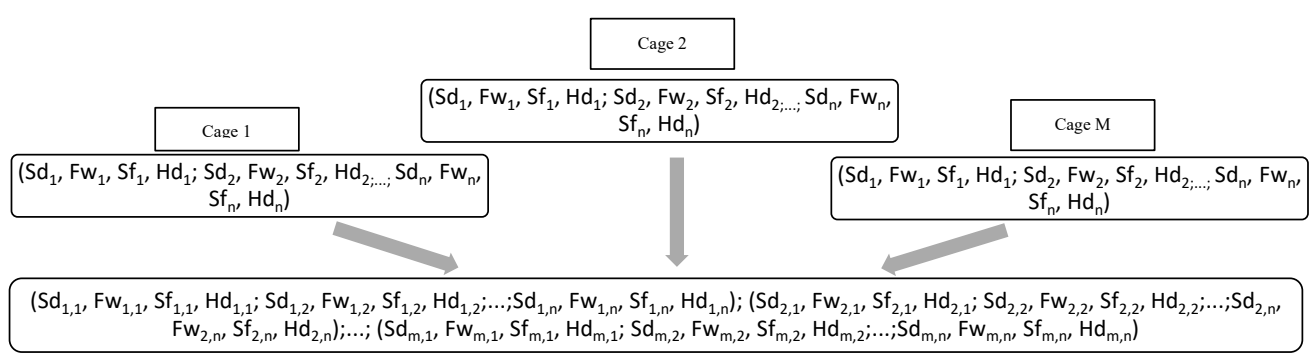

142 In addition to this explanation, in order to facilitate understanding of the methodology developed, section 3 will elucidate the model.

\section{$144 \quad$ 2.1. Multi-criteria model}

145 Given that it is currently necessary to go one step further when attempting to estimate not only 146 profitability, but also results in terms of environmental sustainability and product quality when 147 modelling and simulating in aquaculture, a multi-criteria simulation model was developed. This 148 model allows aquaculture systems to integrate and evaluate the importance of the main criteria 149 that lead decision-makers to select the right strategy for their company.

150 A biological model was defined for this purpose as the basis for three different submodels that simulate the economic, environmental and product quality performance of a farm. To do so, following previous work by Luna et al. (2019a), various criteria were selected within each submodel to represent the most important aspects to consider (Fig. 2). Then, a Multiple-Criteria

154 Decision-Making (MCDM) methodology was used to integrate the simulation of their results in 155 a fitness function that enables the search for an optimal strategic plan. In practice, the producer 156 could choose the most important criteria among those presented here, or even add new ones. 


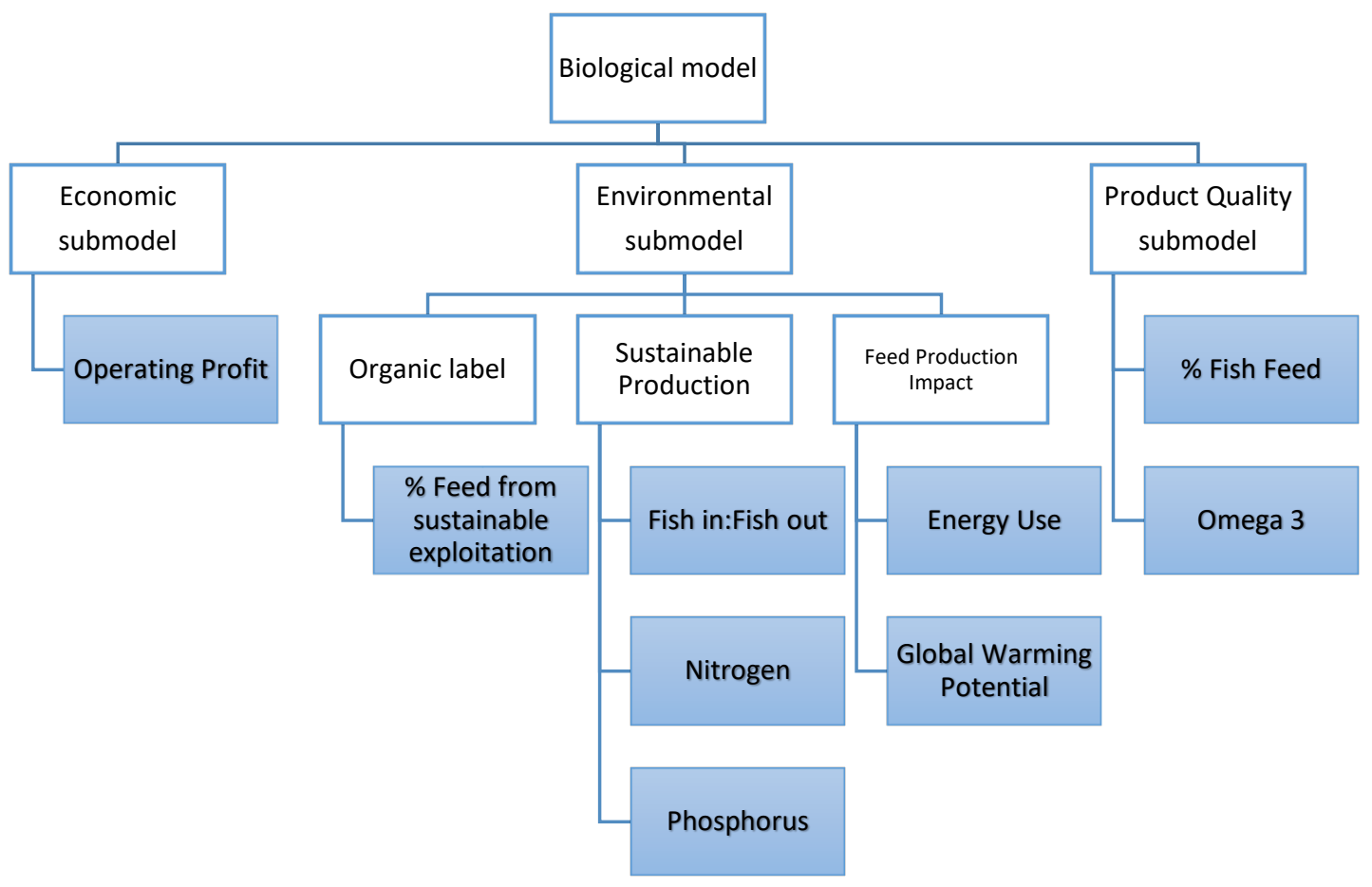

Fig. 2 - Multi-criteria model

In order to apply the MCDM methodology, the Analytic Hierarchy Process (AHP) (Saaty, 1980) was used first to allow producers to rank the criteria according to their importance in order to prioritize the different production alternatives. AHP facilitate this process because it makes it possible to compare alternatives by pairs, forming a matrix that makes it easy to integrate different subjective measures into a final weight for each criterion, turning human judgements into exact or fuzzy numbers (Chan, 2007). Subsequently, as simultaneously optimizing all the criteria is impossible, the objective function to maximize, F(X), is built using the Technique of Order Preference by Similarity to Ideal Solution (TOPSIS). First developed by Hwang and Yoon (1981), this technique estimates the relative closeness, $(d(X))$, of the simulated results to a positive-ideal and a negative-ideal solution for the company based on the relative importance of the criteria.

\subsubsection{Biological model}

The biological model simulates the breeding process, which depends on growth, feeding and mortality rates for the selected production strategy; i.e. in this case, it is based on the seeding date, selected fish fingerlings, feed employed and harvesting date. To do so, the value for each rate depends on three essential factors:

- Water temperature: directly influenced by the seeding and harvesting dates,

- Diet quality: which depends on the selected feed,

- Fish weight: which evolves over time from the initial fingerling weight.

Our model is based on the bioeconomic model described in previous studies by Llorente and Luna $(2013,2014)$. However, it goes one step further, not only because it considers multiple optimization criteria, but also because it starts out from a series of new assumptions that advance the modelling of these processes in aquaculture.

In this regard, the present study has advanced in the practical applicability of these models to aquaculture farming, as it allows multiple cages and production cycles to be considered. This is crucial due to the existing trend in aquaculture of carrying out the fattening process in large 
facilities with the aim of exploiting economies of scale. Furthermore, it enables producers to adapt other decisions, such as those related to feeding, to the company's overall strategy.

In addition, it is currently assumed that the value for growth, feeding and mortality rates depending on these three factors provided by feed suppliers are the correct ones. However, it is also possible to use specific functions based on empirical findings in aspects such us feeding, growth, loss and dispersion according to genetic, source and dietary aspects. The model assumes that there is a range of abiotic factors (temperature, light, salinity and oxygen) which the producer cannot influence in an economically efficient way (Brett, 1979) due to the fact that the process is carried out in sea cages. However, the possibility exists that excessive density in the cage could change how the abiotic factors affect the fish. For this reason, it is assumed that producers will keep the maximum biomass below the maximum insurable biomass density $(20 \mathrm{~kg} / \mathrm{m} 3)$, or at the maximum density allowed in the case of ecolabelled production $\left(15 \mathrm{~kg} / \mathrm{m}^{3}\right)$, so that the main rates are unaffected (Luna, 2002). Therefore, at the seeding date, the number of fingerlings placed in each cage is calculated to obtain the aforementioned biomass density at harvesting time.

Lastly, while other models assume that there are no constraints that may affect the overall seeding and harvesting of the cages, the model developed here assumes the presence of operational and commercial constraints. In the vast majority of cases, all the fish in a cage cannot be harvested at the same time due to labour, physical or commercial constraints; i.e. all the fish from a farm cannot be harvested and sold at the same time. With regard to the seeding date, it is assumed that the offer of fingerlings remains unchanged throughout the year (Gates and Mueller, 1975). Furthermore, it is assumed that all the cages have the same physical characteristics and environmental conditions.

Starting out from those assumptions, the biological model could simulate the growth, feeding and mortality values for each strategy. Based on those results, the developed multi-criteria model includes the following submodels in order to simulate the farm's economic, environmental and quality results.

\subsubsection{Economic submodel}

Although the traditional approach, in which only economic results mattered when designing the aquaculture production strategy, no longer prevails in many cases, these results are still one of the most important outputs for any producer. In this sense, marine aquaculture presents good production times and an acceptable operating margin compared to traditional aquaculture, although profitability varies depending on the decisions taken and a number of external factors.

In the case in hand, the economic model focuses on the maximization of operational profit. This is obtained by subtracting the operating costs incurred in the fattening process from the income obtained from sales.

With regard to operating costs, only variable costs, such as fingerlings and feeding costs, are taken into account, as the remaining costs are not directly influenced by the selected strategy and can be assigned using an allocation key. In particular, feeding costs are the main operating costs in finfish aquaculture and can reach 30-60\% of total production costs (Goddard, 1996).

Income, on the other hand, is calculated as a function of the average mass, its expected dispersion and the market price in USD per kg. This market price for aquaculture produce follows a seasonal pattern for each commercial size of the fish and differs significantly between conventional and 
organic production. Hence, the obtained income will be directly influenced not only by the overall growth achieved, but also by the selected feed and harvesting date.

\subsubsection{Environmental submodel}

The environment is a very important variable in aquaculture, even more so in production processes carried out in sea cages. On the one hand, the biological model analyses how environmental conditions, which cannot be manipulated by the decision maker, affect system performance and should hence be taken into account to make a reliable decision (Casini et al., 2015). However, the effect of the actions carried out throughout the production process on the environment in general and on the surrounding environment in particular is even more important nowadays, hence the need to integrate an environmental submodel.

For this reason, the environmental submodel was divided into different parts that simulate the effect of each of the decisions taken throughout the production process in terms of environmental sustainability:

- First, the origin of the products used as part of the feeding process is taken into account. In this regard, if the producer wishes to apply for an EU Ecolabel, Commission Regulation (EC) No. 889/2008 of 5 September 2008 establishes that feedstuffs shall be fully sourced by-products from organic aquaculture or fisheries certified as sustainable in order to reduce the effect on the environment. This has accordingly been set as a key environmental criterion to include in the model.

- Second, in order to minimize the environmental impact of aquaculture, stakeholders place the highest value on the prevention of nitrogen and phosphorus waste, as well as on increased feed efficiency, measured by the Fish in-Fish out ratio (FIFO) (Lembo et al. (2018)). Hence, the model includes these 3 criteria.

- Lastly, feed production also has an environmental impact and could lead producers to select a different feed or use it in a different way. For this reason, the environmental submodel includes information on energy use (MJ equiv.) and the global warming potential impact $\left(\mathrm{CO}_{2}\right.$ equiv.) of each feeding alternative.

Final values for the above criteria are subsequently estimated in each case based on the information provided by the different feed producers as a percentage of the amount used of each feed.

\subsubsection{Product quality submodel}

The quality of the fish, perceived via its organoleptic characteristics, is directly influenced by many variables ranging from feeding strategies to genetic and environmental factors, including salinity, current and temperature (Rasmussen, 2001; Cordier et al., 2002). However, although it is difficult to find objective criteria that can be easily controlled by the producer in order to increase product quality, the most common representative factor of fish quality is the amount of fatty acids from fatty fish consumed by the farmed fish.

In this regard, some studies Shahidi (2011) refers to the amount of omega-3 fatty acids throughout the entire growth process to optimize fish quality. Otherwise, some studies have shown that it is sufficient for the fish to be fed during the last 90 days with diets containing fish meal and oil to almost fully restore initial fatty acids in muscle (Grigorakis, 2011). Hence, the multi-criteria model includes two criteria to maximize the perception of quality: the use of omega-3 and the fish meal and oil that the feed used during the last 90 days of each batch contain. 


\subsection{Particle swarm optimization process}

270 Given the difficulties of finding an optimal strategy for the problem addressed in this study, namely the complex constraints and the large number of alternatives, classic optimization techniques are not applicable to it or lead to long computation times. Metaheuristic techniques, however, work better under these conditions as they sacrifice the guarantee of finding the optimal solution for the sake of getting good solutions in a significantly reduced amount of time (Blum and Roli, 2003).

Several metaheuristic techniques have been developed in recent years, many of which are inspired by natural processes, such as natural selection for Genetics Algorithms (GA) and swarm intelligence for Particle Swarm Optimizations (PSO). The latter method is especially useful in aquaculture problems like the one addressed in this paper (Cobo et al., 2018), not only because of its advantage in terms of robustness and flexibility, but also due to its higher efficiency when used to solve nonlinear problems with continuous design variables (Hassan et al., 2005).

Furthermore, the problem addressed in this study is sometimes subject to specific conditions. problems, the search space consists of two kinds of points: feasible points, where all the constraints are satisfied; and unfeasible points, where at least one of the constraints is not satisfied (Parsopoulos and Vrahatis, 2002a). In order to solve this problem, PSO allows a Penalty Function to be introduced which solves the $\mathrm{CO}$ problem via a sequence of unconstrained optimization problems (Joines and Houck, 1994).

The PSO methodology developed in the present study follows the steps of the standard particle swarm algorithm initially developed by Kennedy and Eberhart (1995):

1. It starts out by generating a population of random solutions that are distributed in a position, $\mathrm{Xi}(\mathrm{t})$, and moved through the hyperspace with a velocity, $\mathrm{Vi}(\mathrm{t})$.

2. Second, the fitness function is evaluated for those random solutions as the closeness to two hypothetical ideal solutions. In this case, a positive-ideal solution and a negativeideal solution are artificially generated for each situation, as the optimal value for most of the criteria is unknown for the producer.

3. A penalty is then applied to those particles that violate any constraint.

4. At each time step, each particle changes its position due to three components that influence the velocity: the best solution it has achieved $\left(X_{i}^{\text {pbest }}\right)$, the overall best value obtained $\left(X^{\text {best }}\right)$, and an inertia constant $(w)$.

5. Step 3 is repeated until the stopping criterion is met. In the present case, this criterion is the number of movements without any improvement in the fitness function.

Before starting this process, the proper functioning of the PSO algorithm involves choosing the

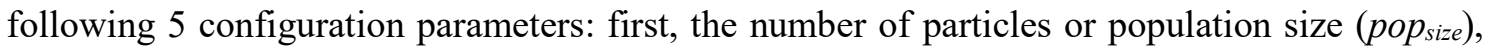
usually set in line with the dimension and the perceived difficulty of the problem (Poli et al., 2007), and the maximum number of iterations; followed by the acceleration coefficients, which are the inertial and the social and personal best positions reached. All these parameters exert a significant influence over the effectiveness of the PSO algorithm and were accordingly selected in a different way for each proposed scenario. In addition, a dynamically modified penalty was set, deducting 1 from the fitness function for each non-satisfied constraint. 


\section{Model description}

\section{Parameters:}

$313 N$, M: maximum number of cages and batches, respectively.

$314 V_{\text {Ool }}$ : capacity $\left(\mathrm{m}^{3}\right)$ of cage $c \in\{1,2, \ldots, N\}$

$315 D o_{\max }, D s_{\max }:$ maximum density of biomass in organic/standard production

$316 N_{\text {weeks }}$ : time horizon (number of weeks)

$317 T_{t}$ : estimated seawater temperature in week $t \in\left\{1,2, \ldots, N_{\text {weeks }}\right\}$

$318 N_{\text {prod }}$ : number of final products. Each product $P_{k}$ with $k \in\left\{1,2, \ldots, N_{\text {prod }}\right\}$ is determined by a

319 species, a type of production (organic/standard) and a minimum commercial size.

$320 N_{\text {feeds }}$ : number of available feeds. Each feed $F_{f}$ with $f \in\left\{1,2, \ldots, N_{\text {feeds }}\right\}$ has the following

321 information: price, $\%$ from sustainable exploitation, residual nitrogen and phosphorus,

322 estimation of the impact of feed production (energy use and global warming potential), $\%$ fish

323 feed and contribution of omega-3.

324 Functions:

$325 M(s, w, T)$ : fish mortality, which depends on the species, its size and water temperature

$326 p_{f}(s, w, p t)$ : fingerling price, as a function of the species, weight and type of production.

$327 p_{d}(w, t, p t)$ : sale price of the final product $d$, which depends on final weight, harvesting time and 328 production type.

$329 f e e d Q(f, p)$ : a Boolean function that determines whether feed $F_{f}$ is suitable for the production of 330 product $P_{p}$.

$331 R_{f}\left(w, T_{t}\right)$ : food ration of feed $F_{f}$, which depends on fish weight and water temperature.

$332 G R_{f}\left(w, T_{t}\right)$ : growth rate of the fish using feed $F_{f}$, which depends on fish weight and water 333 temperature.

\section{Decision variables:}

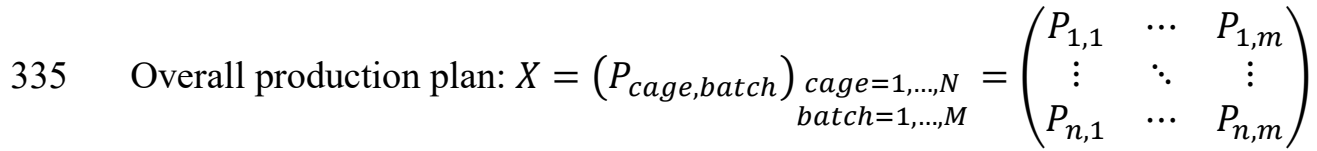

336 Planning the production of a batch from a cage:

$$
P_{\text {cage }, \text { batch }}=\left(S d_{\text {cage }, \text { batch }}, P t_{\text {cage }, \text { batch }}, F w_{\text {cage }, \text { batch }}, F_{\text {cage }, \text { batch }}, H d_{\text {cage }, \text { batch }}\right)
$$

338 where 
$H d_{\text {cage,batch }} \in\left\{1,2, \ldots, N_{\text {weeks }}\right\}:$ harvesting date (week number from the initial week, never before reaching the minimum commercial weight)

Particle Swarm Optimization algorithm:

pop $_{\text {size: }}$ population size (number of particles)

$w \in[0,1]:$ inertia component weight

$\alpha, \beta \in[0,1]$ : social and personal best component weights $X_{i}^{k}$ and $V_{i}^{k}$ with $1=1, \ldots$, pop $_{\text {size }}$ and $k=1, \ldots$, iter $r_{\text {max }}$ : position and velocity of particle $i$ in iteration $k$.

$X^{\text {best }}$ : global best position during the process, according to the fitness function

$V_{i}^{k}=w V_{i}^{k}+\alpha \operatorname{rand}(0,1)\left(X^{\text {best }}-X_{i}^{k}\right)+\beta \operatorname{rand}(0,1)\left(X_{i}^{\text {pbest }}-X_{i}^{k}\right)$ : velocity vector for particle $i$ in iteration $k$.

$356 X_{i}^{k+1}=X_{i}^{k}+V_{i}^{k}$ : update of particle positions

Fitness function (proximity to ideal solution):

$C_{j}\left(X_{i}^{k}\right) \quad j=1, \ldots, 9:$ normalized values of the decision criteria in each particle

$d^{+}\left(X_{i}^{k}\right)$ : distance from the positive ideal solution of criteria values of particle $i$.

$d^{-}\left(X_{i}^{k}\right)$ : distance from the anti-ideal solution of criteria values of particle $i$.

$361 F\left(X_{i}^{k}\right)=\frac{d^{-}\left(X_{i}^{k}\right)}{d^{-}\left(X_{i}^{k}\right)+d^{+}\left(X_{i}^{k}\right)}-$ Penalty $\left(X_{i}^{k}\right)$ : relative closeness of particle with respect to ideal

362 solution with a penalty if constraints are violated.

Objective: maximize the fitness function $F(X)$.

\section{Constraints:}

$\operatorname{minp}(w) \leq \operatorname{Prod}_{w}\left(X_{i}^{k}\right) \leq \operatorname{maxp}(w)$ with $w=1, \ldots, N_{\text {weeks }}:$ commercial or operational constraints for week $w$.

where

$\operatorname{Prod}_{w}(X)=\sum_{\text {cage }=1}^{N}$ Harvest $_{X}($ cage, $w)$ : this represents the sum of amounts harvested in week $w$ according to plan $X$.

\section{Results}

As an example of practical application, the developed methodology was applied to the decisionmaking process of a hypothetical aquaculture farm. In the present case, the information required to define the hypothetical farm comes both from primary sources, such as oceanographic buoys and feed manufacturers, and to a lesser extent from secondary sources, namely other research studies. 
The simulation and optimization process takes place in two consecutive steps: first, the estimation of the objective function, based on the multi-criteria model; followed by the use of the PSO methodology to find a near optimal strategy that maximizes the overall results of the farm. To this end, each cage at the farm adopts a synchronized strategy that consists of the seeding date, harvesting date, feeding alternative and selected fish fingerling, for all its cycles.

However, before starting, each decision variable is limited by the internal characteristics of the farm and the underlying assumptions:

- Characteristics of the farm: A gilthead seabream farm with several cages was simulated based on common characteristics of Mediterranean sea farms. The proposed objective was the optimization of production for a farm with 3 different cages over a two-year horizon (Table 1). It will thus be possible to carry out a maximum of two production cycles, which cannot be extended beyond the given end date. All the cages have a capacity of $200 \mathrm{~m}^{3}$, although the maximum biomass density in each one will depend on the type of production selected, as the maximum usually applied is $20 \mathrm{Kg} / \mathrm{m}^{3}$. In the case of organic production, this maximum would decrease to $15 \mathrm{~kg} / \mathrm{m}^{3}$.

\begin{tabular}{cc} 
Parameter & Value \\
\hline Starting Date & $17 / 06 / 2019$ (Week 0) \\
End Date & $14 / 06 / 2021$ (Week 104) \\
Number of cages & 3 \\
Cage capacity & $200 \mathrm{~m}^{3}$ \\
Feasible harvest & $(300,1000) \mathrm{g}$ \\
sizes & Tarragona (2720)
\end{tabular}

Table 1 - Farm characteristics.

- Farmed fish: Although this methodology allows farms to make the decision regarding which type (weight and species) of fingerlings to seed in each cage, it is not realistic to expect two completely differentiated products in such a small farm. Furthermore, the feeding decision already allows combining two types of production, organic and traditional. Hence, we proceed in this case under the assumption that each cage starts out with gilthead seabream fingerlings weighing $30 \mathrm{~g}$ on a date to be determined.

- Feeding decision: Three different feedstuffs were included as a representation of a number of different feeding alternatives within the feed market. In all three cases, data on feeding, growth and mortality rates and feed components were provided directly by the feed producer. With regard to feed production criteria, these were estimated based on a secondary source, the study conducted by Pelletier and Tyedmers (2007), which approximates their values depending on the feed ingredients. In this regard, the first feed (F1) represents a normal feed, with acceptable rates under normal circumstances and a very competitive price. The second (F2) is a feed with an increased percentage of fish protein, which means better growth rates even under unfavourable weather conditions, but it has a slightly higher price. The third feed (F3) represents the choice of organic production, as it is a high quality, high price feed made entirely with products from organic fisheries/production.

- Producer preferences: Lastly, the present study assumes that the producer affords more importance to the economic performance of the farm, as this is the traditional and most common preference of aquaculture producers with respect to the importance of the criteria under study here (Table 2). To this end, the criteria were compared by pairs, using 
the MCDM technique to assign a specific weight to each one. The economic criterion was thus found to be the most important one, although the criteria of efficiency (fish infish out ratio) and omega-3 (which affects quality) are also taken into consideration (Luna et al., 2019a).

In addition, from the very beginning and throughout the entire production process, many external factors directly influence the results obtained for each candidate solution and hence the final plan selected. First, the main variable affecting the biological model is the water temperature. In this respect, the Mediterranean Sea is the most common place to farm gilthead seabream and so it was chosen as the hypothetical location for the farm. The annual information on temperature was obtained from the Spanish Port Authority's network of oceanographic buoys in a location close 433 to Tarragona (Fig. 3).

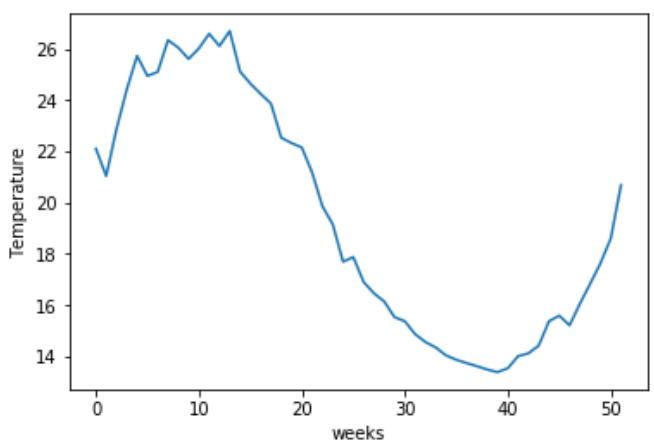

Fig. 3 - Average Farm Temperature

\begin{tabular}{cc} 
Criteria & Scenario 1 \\
\hline Economic Criteria & $\mathbf{8 1 . 8 \%}$ \\
Profit & \multicolumn{2}{c}{$81.8 \%$} \\
Environmental Criteria & $\mathbf{9 . 1 \%}$ \\
\% Organic Feed & $0.3 \%$ \\
Fish in-Fish out Ratio & $3.2 \%$ \\
Total Nitrogen & $1.0 \%$ \\
Total Phosphorus & $1,0 \%$ \\
Energy Use & $1.8 \%$ \\
Global Warming Potential & $1.8 \%$ \\
Quality Criteria & $\mathbf{9 . 1 \%}$ \\
\% Fish origin feed & $0.9 \%$ \\
Omega-3 & $8.2 \%$ \\
Table 2 - Producer preferences.
\end{tabular}


setting an ideal alternative (which will never be reached) for each of the criteria as the objective and measuring the fulfilment of this objective via the fitness function.

In addition, like other metaheuristic techniques, Particle Swarm Optimization, is distinguished by its capacity to find an optimal solution (unknown until that moment) for complex, real-world problems. Therefore, the ideal or anti-ideal solutions have not been found prior to running the PSO algorithm, and they are probably not found in any case. For this reason, the developed methodology includes an initial step in which the hypothetical positive-ideal and negative-ideal solutions are generated artificially (Luna et al., 2019b). To do so without incurring a high computational cost, a hypothetical solution is generated each time whose aim is to exploit the full potential of the farm; i.e. seeding as soon as possible and harvesting on the last day for each feed alternative. This hypothetical solution is then multiplied by a supplement of $\pm 75 \%$, assuming that the PSO can find an alternative with better results, but not as good as $75 \%$ better.

In the present case, the results shown in Table 3 were found in the initial step and "+ideal" and "ideal" were estimated from these results as explained previously.

Criteria

Obj
F1

F2

\begin{abstract}
F3
\end{abstract}
- ideal

\section{Economic Criteria}

Profit (\$)

MAX $\quad 55,856$

56,182

49,358

98,318

12,339

\section{Environmental Criteria}

Organic Feed (\%)

MAX

$$
0 \%
$$

$$
0 \%
$$

$100 \%$

$100 \%$

0\%

Fish in-Fish out Ratio

MIN

$48 \%$

$70 \%$

$91 \%$

$12 \%$

$160 \%$

Total N (g)

MIN

$3.49 \mathrm{E}+06$

$3.34 \mathrm{E}+06$

$3.03 \mathrm{E}+06$

757,949

6.11E+06

Total P (g)

MIN

733,872

762,136

535,924

133,981

$1.33 \mathrm{E}+06$

Energy Use (MJ equiv.)

MIN

$4.38 \mathrm{E}+08$

$2.14 \mathrm{E}+08$

$3.80 \mathrm{E}+08$

$5.34 E+07$

$7.66 \mathrm{E}+08$

Global Warming $\left(\mathrm{kg} \mathrm{CO}_{2}\right.$ equiv.)

\section{MIN $\quad 3.84 \mathrm{E}+07 \quad 3.87 \mathrm{E}+07$}

$1.22 \mathrm{E}+07$

$3.06 \mathrm{E}+06$

6.77E+07

\section{Quality Criteria}

$$
\% \text { Fish origin feed }
$$

Omega-3 (\%)
460

MAX $\quad 24 \%$

MAX $\quad 0.98 \%$

$$
37 \%
$$

$0.98 \%$

$$
54 \%
$$$$
94 \%
$$$$
6.1 \%
$$

$1.96 \%$
$0.24 \%$

Table 3 - Hypothetical alternatives

Especial attention should be drawn to the fact that the multi-criteria model stands out as the most important part of the methodology, as both the initial step of estimating the results in order to generate the optimization objective and the evaluation of each alternative found by each particle of the PSO involves the use of the model. As explained previously, it first estimates the achieved growth and the amount of feed used on a daily basis and then the submodel used to estimate the value of each criterion is calculated from these data.

\subsection{Selection of the optimal strategic plan}

In addition to the above explanation of all that is needed to test the developed methodology, there are two other constraints that should be included in order to test the method in the most appropriate way, namely operational and the commercial constraints. These should be included because their 
existence is inevitable in companies of this type, although including them also complicates the search for useful solutions.

Accordingly, the search for a near optimal strategic plan was tested under the following three theoretical scenarios involving optimization constraints:

\subsubsection{Unrestricted production}

First, the developed methodology was tested in a scenario without any operational or commercial constraints. This enables the proper functioning of the methodology to be tested in a situation in which every candidate solution within the search space constitutes a valid alternative. This facilitates the process and means that the number of particles and interactions can be lower. In this scenario, the five parameters of the PSO algorithm were as follows: the population was 90 particles with a maximum number of iterations of 30 , while the inertia, cognitive and social components each took the value of 0.5. Appropriate parameters selection is a fundamental aspect of PSO and it is discussed further in Section 5.

Table 4 shows how, when no constraints force the different cages to adapt to each other, all the three cages tend to choose the same strategy, which we assume to be optimal: harvesting in the same week and taking the same feeding decision. Together with the practicality of the selected strategies, as we will see later, this suggests the proper functioning of the methodology right from the start. As an exception, it is possible to see small differences in some points that would undoubtedly be solved with more computing time.

\begin{tabular}{ccccc}
\hline & Results & Cage 1 & Cage 2 & Cage 3 \\
\hline \multirow{4}{*}{ Cycle 1 } & Seeding week & 2 & 3 & 4 \\
& Harvesting week & 33 & 33 & 39 \\
& Feed & F3 & F3 & F3 \\
& Seabream & 30 & 30 & 30 \\
& Fingerling Weight & & & 46 \\
& Seeding week & 42 & 42 & 86 \\
& Harvesting week & 86 & 86 & F1 \\
& Feed & F2 & F2 & 30 \\
\hline & Seabream & 30 & 30 & \\
\hline \multicolumn{2}{c}{ Closeness: 0.62 } \\
\hline
\end{tabular}

Table 4 - Candidate solution 1

\subsubsection{Weekly constraints on maximum production}

In the second scenario, two different constraints affecting the maximum volume of fish harvested per week were added:

- Operational: In practice, operational constraints on farms, such as their labour capacity, reduce their decision-making capacity. For this reason, it is more realistic to take into account the impossibility of harvesting an entire cage in the selected week, forcing the model to consider the harvesting time to last at least 1 month (4 weeks).

- Commercial: In addition, it is not a good idea for the company to saturate the market in a specific week, thus lowering the selling prices. In order to avoid this situation, a maximum of 4 tons per month (1T/Week) was fixed.

In this case, there are some candidate solutions that do not meet the requirements. In order to ensure compliance with the constraints without losing optimization capacity, a penalty function was defined to transform this Constrained Optimization (CO) problem into an unconstrained one. 
This way, no further changes in the optimization parameters were needed to find an equally valid 505 solution.

\begin{tabular}{ccccc}
\hline & Results & Cage 1 & Cage 2 & Cage 3 \\
\hline \multirow{4}{*}{ Cycle 1 } & Seeding week & 3 & 3 & 4 \\
& Harvesting week & 35 & 30 & 39 \\
& Feed & F3 & F3 & F3 \\
& Seabream & 30 & 30 & 30 \\
& Fingerling Weight & & & 46 \\
Cycle 2 & Seeding week & 44 & 38 & 85 \\
& Harvesting week & 89 & 81 & F1 \\
& Feed & F1 & F2 & 30 \\
\hline & Fingerling Weight & 30 & 30 & 30 \\
\multicolumn{2}{c}{ Closeness: 0.61 }
\end{tabular}

Table 5 - Candidate solution 2

As can be seen from Table 5, these constraints have forced the methodology to find a strategy that splits the harvesting process, leaving a month between each cage. Furthermore, the cage is now harvested over the four following weeks.

\subsubsection{Weekly constraints on minimum production}

Lastly, the capacity of the developed PSO algorithm to obtain good results in even more complex $\mathrm{CO}$ problems is tested. With this aim in mind, a minimum volume of harvested fish on specific dates in order to comply with commercial commitments was also included, in addition to the aforementioned constraints.

Specifically, it is assumed that the farm agreed to sell 0.5 Tons of gilthead seabream weighing around $300 \mathrm{~g}$ in the following four weeks: $30,50,70,90$. This constraint forces the methodology to find a strategy in which there are not two different point in which all the cages are harvested, but rather that the process is carried out in a more distributed way. This would allow the company to obtain profits in a sustained manner throughout the year, but it also makes the problem much more complex.

In this scenario, there are a vast majority of regions of the search space where the constraints are not met. This new situation forces us to increase the number of particles to 120 , thus covering a larger area, since the algorithm may sometimes not find a feasible solution if only 90 particles are used. Those issues are discussed further in the next section.

Finally, these constraints were met and the harvesting dates shifted to separate areas (Table 6).

\begin{tabular}{ccccc}
\hline & Results & Cage 1 & Cage 2 & Cage 3 \\
\hline \multirow{4}{*}{ Cycle 1 } & Seeding week & 0 & 2 & 5 \\
& Harvesting week & 28 & 48 & 38 \\
& Feed & F3 & F3 & F2 \\
& Seabream & 30 & 30 & 30 \\
& Fingerling Weight & & & \\
& Seeding week & 34 & 55 & 47 \\
Cycle 2 & Harvesting week & 70 & 84 & 88 \\
& Feed & F2 & F2 & F2 \\
& Seabream & 30 & 30 & 30 \\
\hline \multicolumn{2}{c}{ Closeness: 0.55 } \\
\hline
\end{tabular}


Figure 4 shows in graphic form how a different strategy was obtained in each of the verification scenarios described above. The third scenario is particularly worth highlighting, in which four mandatory points of sale are established, forcing the displacement of the optimal points.

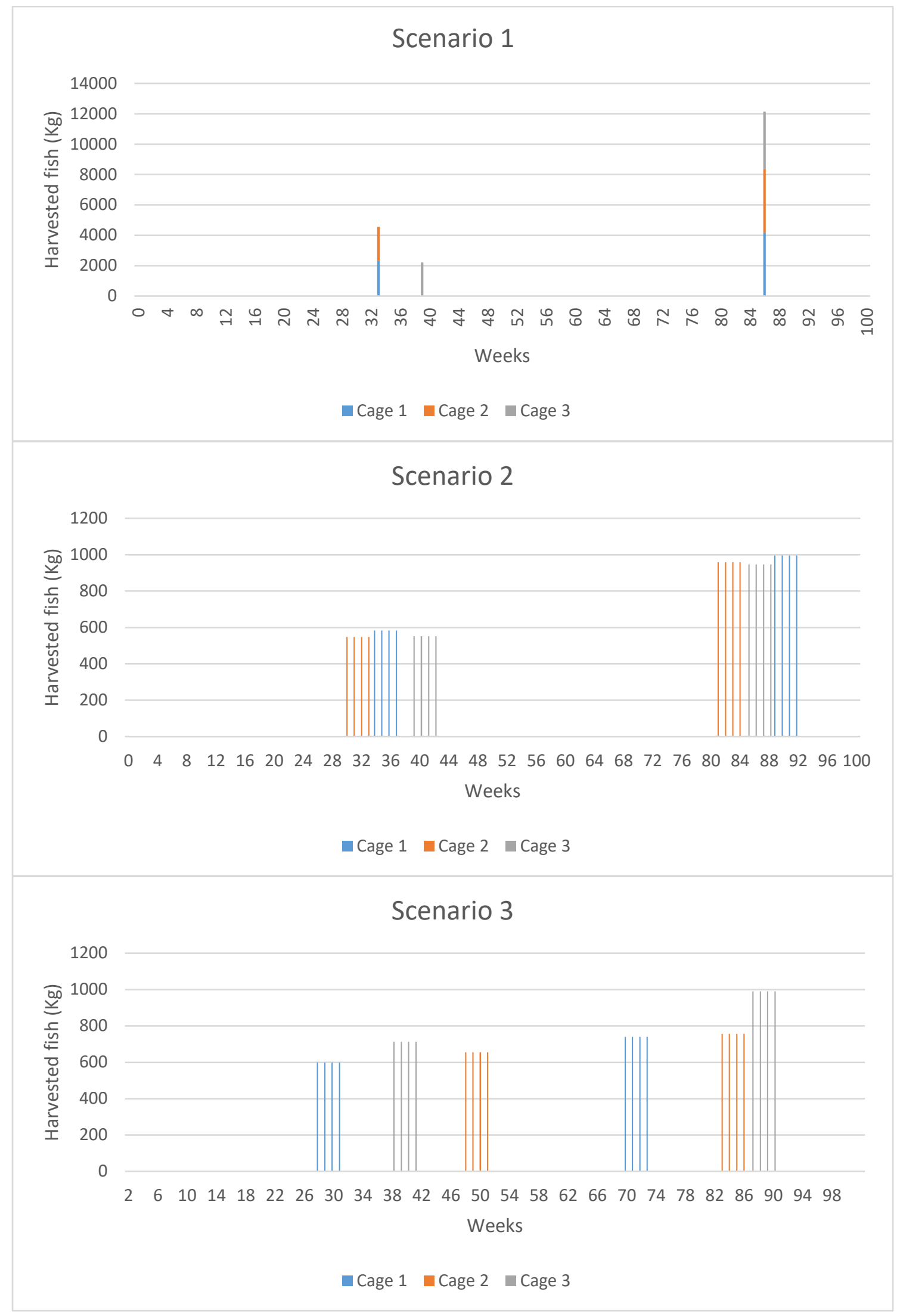


Regarding the results thus obtained, profits decrease with increasing operational or commercial constraints, as expected. This is explained by the limitation that the system receives when looking for an optimal alternative. However, in all three scenarios, both positive profits and better-thanexpected environmental and quality results are obtained (Table 7), as they were based on the alternatives artificially created in the previous section.

Scenario 1

Scenario 1 Scenario 2 Scenario 3

\begin{tabular}{cccc}
\hline $\begin{array}{c}\text { Economic Criteria } \\
\text { Profit (\$) }\end{array}$ & 65,892 & 65,165 & 60,120 \\
Environmental Criteria & & & \\
Organic Feed (\%) & $42 \%$ & $41 \%$ & $34 \%$ \\
Fish in-Fish out Ratio & $54 \%$ & $46 \%$ & $56 \%$ \\
Total N (g) & $2.63 \mathrm{E}+06$ & $2.59 \mathrm{E}+06$ & $2.31 \mathrm{E}+06$ \\
Total P (g) & 537,257 & 511,169 & 485,508 \\
Energy Use (MJ equiv.) & $2.01 \mathrm{E}+08$ & $2.98 \mathrm{E}+08$ & $1.35 \mathrm{E}+08$ \\
Global Warming (kg CO ${ }_{2}$ equiv.) & $2.71 \mathrm{E}+07$ & $2.61 \mathrm{E}+07$ & $2.43 \mathrm{E}+07$ \\
Quality Criteria & & & \\
\% Fish origin feed & $42 \%$ & $37 \%$ & $43 \%$ \\
$\quad$ Omega-3 (\%) & $1.39 \%$ & $1.39 \%$ & $1.31 \%$
\end{tabular}

Table 7 - Results from each

\section{Discussion and conclusions}

Over the course of the past few decades, aquaculture has established itself as a flagship industry in the agri-food sector, mainly due to advances in intensive production methods and its longerterm advantage in terms of environmental sustainability. However, while other industries have greatly improved their management capacity, decision-making in aquaculture is still very complex due to biological, technical and environmental factors. In this regard, several studies have addressed this problem using bio-economic models and techniques to better understand and optimize decision-making processes in aquaculture (Llorente and Luna, 2016; Besson et al., 2016). However, there is still a need for improvements that take into account new social requirements in terms of environmental sustainability and product quality.

Aquaculture currently faces new challenges due to changes in fish production and consumption patterns. Stakeholders demand more and better fish, but also more pro-environmental behaviour on the part of farms. To meet these demands in a cost-effective way, companies should increase the efficiency of their production process, farming fish intensively in large facilities with multiple cages and an organized plan for long-term farming. This creates an urgent need for technical assistance to address the strategic decision-making process, optimizing the value of multiple objectives at a fish farm with multiple batches, cages, feedstuffs and products.

To address this problem, a methodology that integrates a multi-criteria model and a Particle Swarm Optimization (PSO) technique has been developed and tested in this paper. The results have shown the great capacity of the developed methodology for both simulating the fattening 
process at an aquaculture farm regarding multiple criteria and finding near-optimal solutions in 558 different scenarios. This will substantially improve the management capacity of fish producers, more necessary than ever before due to the demands of various stakeholders and high market 560 competitiveness.

561 As to the multi-criteria model developed in the paper, this has enabled us to systematically link 562 the economic, environmental and quality results of aquaculture farms with their biological 563 performance. This approach has enabled the methodology to achieve the goal of overcoming 564 central aquaculture-specific constraints and gaps in this field, such as the integration of several cages and cycles in a synchronized strategic plan. Furthermore, the possibility of considering new ways of production, with their own legal requirements in terms of feed ingredients or maximum stocking density, constitutes another advantage, mainly in terms of adapting to the new ecological global trend. These improvements have been directly pointed out in many previous studies, highlighting the complexity of integrating more than one cage or production unit (Llorente and Luna, 2014) and the absence of well-documented multi-criteria systems for aquaculture (Mathisen, 2016)

Furthermore, the decision to consider operational and commercial constraints has meant an added difficulty when addressing the problem of decision-making in aquaculture. However, it has proven to be a well-founded decision, as the existence of labour and market constraints regarding maximum weekly production is inevitable in this sector. In addition, having commercial agreements on specific dates has been shown to have a major effect on the company's decisions, both due to the impossibility of complying with them on certain dates and because they could lead to a reduction in profit. Nonetheless, they represent a reduction in the uncertainty surrounding company sales, which is very important in a risk sector such as aquaculture.

580 With respect of the optimization process, the Particle Swarm Optimization (PSO) method is a swarm intelligence method that models social behaviour to guide swarms of particles towards the most promising regions of the search space (Eberhart and Kennedy, 1995). This method has a proven capacity to deal efficiently with Multiobjective Optimization (MO) problems, which are very common due to the multi-criteria nature of most real-world problems (Parsopoulos and Vrahatis 2002b). In the present study, PSO confirmed its capacity once again, obtaining good results for the company not only in traditional MO problems, but also in complex Constrained Optimization (CO) problems, including those in which both commercial and operational constraints coexist.

589 The development of this methodology directly addresses one of the key challenges in aquaculture in recent years, the ultimate goal of which is to improve efficiency in order to minimize the use of resources and maximize profits. However, the inclusion of those multiple, complex constraints increases the complexity that the optimization methodology has to face and hence the computational cost of the entire process. Hence, another crucial point of discussion in the present study, like in most PSO applications, is the selection of suitable method specifications in order to optimize the trade-off between exploration and exploitation, thereby increasing the efficiency of this search for optimal strategies.

597 The first decision in this regard should be about how to ensure compliance with the constraints 598 without losing optimization capacity. The most common approach for solving CO problem is the use of a penalty function to transform a constrained problem into an unconstrained one. Penalty values can be fixed throughout the minimization (stationary penalty function) or dynamically modified (non-stationary penalty function), although results obtained using the latter are almost always superior (Parsopoulos and Vrahatis, 2002a). In order to choose the best possible solution to this problem, three alternatives have been compared 10 times, applying the parameters initially established (90 particles with a maximum number of iterations of 30$)$ : 
- A strategy in which the closeness of every candidate solution that does not meet all the constraints is automatically changed to 0 .

- A stationary penalty function that subtracts one (-1) from the closeness if any constraint is not met.

- A strategy in which the penalty is dynamically modified, subtracting one (-1) by each violated constraint.

As can be seen in the Table 8, the third strategy also proved to be the best alternative in this case. However, this strategy is not sufficient enough to address this complex problem efficiently.

\begin{tabular}{cccc}
\hline Method & Best Solution & Mean Solution & $\begin{array}{c}\text { \% of cases it founds } \\
\text { a feasible solution }\end{array}$ \\
\hline Closeness 0 & 0.36 & 0.16 & $60 \%$ \\
Fixed -1 & 0.51 & 0.25 & $60 \%$ \\
Dynamic & 0.55 & 0.43 & $90 \%$ \\
\hline
\end{tabular}

Table 8 - Penalty function comparison

In addition to the above, with the same aim, the importance of a convenient combination of the five PSO parameters is much higher in constrained optimization problems. On the one hand, increasing the number of solutions that need to be tested could be an option, although reducing waiting times and making better use of this method is also a primary objective. Therefore, there is an initial need to choose between two options regarding these parameters: solving the most complex problems by having a large population of particles, or moving the particles around in the search-space more times.

On the other hand, there is another way of addressing the challenge of balancing the trade-off between exploration and exploitation via the three components that influence the movements of particles in order to require fewer iterations on average to find the optimum solution. In this regard, Shi and Eberhart (1998) showed how, for example, a larger inertia weight facilitates global exploration (searching new areas), while a smaller inertia weight tends to facilitate local exploitation of the current search area. Similarly, the balance between the importance of the best solution that a particle has achieved (pbest) and the overall best value obtained (gbest) can also vary these "exploration abilities".

As explained in Section 2, in the present study we chose to focus on testing the multi-criteria model and PSO capacity to find a useful solution, Hence, starting out from a larger population of particles in order to cover more search-space was found to be sufficient to address even the constrained problems, as can be seen in the Table 9 .

\begin{tabular}{cccc}
\hline Particles & Best Solution & Mean Solution & $\begin{array}{c}\text { \% of cases it founds } \\
\text { a feasible solution }\end{array}$ \\
\hline 60 & 0.41 & 0.20 & $50 \%$ \\
90 & 0.55 & 0.43 & $90 \%$ \\
120 & 0.58 & 0.50 & $100 \%$ \\
\hline
\end{tabular}

Table 9 - Number of particles

Results achieves illustrate that the proposed strategic plan thus achieved a good economic profits in all the three scenarios while also taking all the other variables into consideration. We may conclude that this methodology will improve the management capacity of aquaculture producers and their understanding of the performance of the main variables of the farm. Furthermore, any effort aimed at increasing information recording and transparency will improve these results.

The process of determining the suitable combination of parameters stands out as a future line of research in order to validate and improve the efficiency and applicability of this methodology. This would require either preliminarily optimizing all of them at the same time, which requires a 
642 high computational capacity to do so, or introducing a methodology for dynamic or self-adaptive 643 parameters, which have proven to be an option that obviates this tedious pre-processing task of 644 parameter fine-tuning (Montalvo et al., 2010). 
1- Besson, M., Vandeputte, M., van Arendonk, J., Aubin, J., de Boer, I., Quillet, E., \& Komen, H. (2016). Influence of water temperature on the economic value of growth rate in fish farming: The case of sea bass (Dicentrarchus labrax) cage farming in the Mediterranean. Aquaculture, 462, 47-55. doi: 10.1016/j.aquaculture.2016.04.030

2- Blum, C., \& Roli, A. (2003). Metaheuristics in combinatorial optimization. ACM Computing Surveys, 35(3), 268308. doi: 10.1145/937503.937505

3- Brett, J.R. (1979). Environmental factors and growth. Fish Physiology 8, 599-675. doi: 10.1016/S15465098(08)60033-3.

4- Casini, M., Mocenni, C., Paoletti, S., \& Pranzo, M. (2015). Decision support system development for integrated management of European coastal lagoons. Environmental Modelling \& Software, 64, 47-57. doi: 10.1016/j.envsoft.2014.11.008

5- Chan, F., \& Kumar, N. (2007). Global supplier development considering risk factors using fuzzy extended AHPbased approach. Omega, 35(4), 417-431. doi: 10.1016/j.omega.2005.08.004

6- Cobo, A., Llorente, I., \& Luna, L. (2015). Swarm Intelligence in Optimal Management of Aquaculture Farms. In: Handbook of Operations Research in Agriculture and the Agri-Food Industry. Springer.

7- Cobo, A., Llorente, I., Luna, L., and Luna, M. (2018). A decision support system for fish farming using particle swarm optimization. Computers and Electronics in Agriculture, DOI: 10.1016/j.compag.2018.03.036.

8- Cordier, M., Brichon, G., Weber, J., \& Zwingelstein, G. (2002). Changes in the fatty acid composition of phospholipids in tissues of farmed sea bass (Dicentrarchus labrax) during an annual cycle. Roles of environmental temperature and salinity. Comparative Biochemistry And Physiology Part B: Biochemistry And Molecular Biology, 133(3), 281-288. doi: 10.1016/s1096-4959(02)00149-5

9- Dapueto, G., Massa, F., Costa, S., Cimoli, L., Olivari, E., \& Chiantore, M. et al. (2015). A spatial multi-criteria evaluation for site selection of offshore marine fish farm in the Ligurian Sea, Italy. Ocean \& Coastal Management, 116, 64-77. doi: 10.1016/j.ocecoaman.2015.06.030

10- de Campos, A., Pozo, A., \& Duarte, E. (2019). Parallel multi-swarm PSO strategies for solving many objective optimization problems. Journal Of Parallel And Distributed Computing, 126, 13-33. doi: 10.1016/j.jpdc.2018.11.008

11- Eberhart, R. C., and Kennedy, J. (1995). A new optimizer using particle swarm theory, Proc. Sixth Intl. Symp. on Micro Machine and Human Science (Nagoya, Japan), IEEE Service Center, Piscataway, NJ, 39-43.

12- European Commission, 2008. Commission Regulation No 889/2008 of 5 September 2008 laying down detailed rules for the implementation of Council Regulation (EC) No 834/2007 on organic production and labelling of organic products with regard to organic production, labelling and control. Including amendments. http://eurlex.europa.eu/legal-content/EN/TXT/?qid=1515078877100\&uri=CELEX:02008R0889-20180101

13- Føre, M., Frank, K., Norton, T., Svendsen, E., Alfredsen, J., \& Dempster, T. et al. (2018). Precision fish farming: A new framework to improve production in aquaculture. Biosystems Engineering, 173, 176-193. doi: 10.1016/j.biosystemseng.2017.10.014

14- Gates, J.M., Mueller, J.J., 1975. Optimizing the growth and marketing of fish in a controlled environment. Mar. Technol. 9 (5), 13-16.

15- Goddard, S. (1996) Feed Management in Intensive Aquaculture. Chapman \& Hall, New York, USA.

16- Granada, L., Lopes, S., Novais, S., \& Lemos, M. (2018). Modelling integrated multi-trophic aquaculture: Optimizing a three trophic level system. Aquaculture, 495, 90-97. doi: 10.1016/j.aquaculture.2018.05.029

17- Grigorakis, K. (2010). Effects of Nutrition and Aquaculture Practices on Fish Quality, in: Alasalvar, C., Shahidi, F., Miyashita, K., Wanasundara, U. (Eds.), Handbook of Seafood Quality, Safety and Health Applications. WileyBlackwell, 82-95

18- Hassan, R., Cohanim, B., de Weck, O., Venter, G., 2005. A comparison of particle swarm optimization and the genetic algorithm. In: 46th AIAA/ASME/ASCE/AHS/ASC Structures, Structural Dynamics and Materials Conference, Structures, Structural Dynamics, and Materials and Co-located Conferences.

19- Hwang, C.L. \& Yoon, K. (1981) Multiple Attribute Decision Making: Methods and Applications. SpringerVerlag, New York. http://dx.doi.org/10.1007/978-3-642-48318-9

20- Ishizaka, A., \& Labib, A. (2011). Review of the main developments in the analytic hierarchy process. Expert Systems with Applications, 38(11), 14336-14345. https://doi.org/10.1016/j.eswa.2011.04.143

21- Joines, J.A. \& Houck, C.R. (199\$): On the Use of Non-Stationary Penalty Functions to Solve Nonlinear Constrained Optimization Problems with GA's. Proc. IEEE Int. Conf. Evol. Comp., 579-585.

22- Lembo, G., Jokumsen, A., Spedicato, M., Facchini, M., \& Bitetto, I. (2018). Assessing stakeholder's experience and sensitivity on key issues for the economic growth of organic aquaculture production. Marine Policy, 87, 8493. doi: 10.1016/j.marpol.2017.10.005

23- Llorente, I., \& Luna, L. (2013). The Competitive Advantages Arising from Different Environmental Conditions in Seabream,Sparus aurata, Production in the Mediterranean Sea. Journal Of The World Aquaculture Society, 44(5), 611-627. doi: 10.1111/jwas.12069 
24- Llorente, I., \& Luna, L. (2013). The Competitive Advantages Arising from Different Environmental Conditions in Seabream,Sparus aurata, Production in the Mediterranean Sea. Journal Of The World Aquaculture Society, 44(5), 611-627. doi: 10.1111/jwas.12069

25- Llorente, I., \& Luna, L. (2014). Economic optimisation in seabream (Sparus aurata) aquaculture production using a particle swarm optimisation algorithm. Aquaculture International, 22(6), 1837-1849. doi: 10.1007/s10499-0149786-2

26- Llorente, I., \& Luna, L. (2016). Bioeconomic modelling in aquaculture: an overview of the literature. Aquaculture International, 24(4), 931-948. doi: 10.1007/s10499-015-9962-z

27- Luna, L. (2002). Economic analysis of finfish mariculture operations in Spain. Aquaculture Economics \& Management, 6(1-2), 65-79. doi: 10.1080/13657300209380304

28- Luna, M., Llorente, I. \& Cobo, A. (2019a). Determination of feeding strategies in aquaculture farms using a multiple-criteria approach and genetic algorithms. Annals of Operations Research. DOI: 10.1007/s10479-01903227-w

29- Luna, M., Llorente, I. \& Cobo, A. (2019b). Integration of environmental sustainability and product quality criteria in the decision-making process for feeding strategies in Seabream aquaculture companies. Journal of Cleaner Production 217:691-701. DOI: 10.1016/j.jclepro.2019.01.248

30- MAPAMA (2012). Estudio de la cadena de valor y formación de precios de la Dorada de acuicultura. Observatorio de Precios de los Alimentos, Madrid, Spain.

31- Mardle, s., \& pascoe, s. (1999). A Review of Applications of Multiple-Criteria Decision-Making Techniques to Fisheries. Marine Resource Economics, 14(1), 41-63. doi: 10.1086/mre.14.1.42629251

32- Mathisen, B.M., Haro, P., Hanssen, B., Björk, S. \& Walderhaug, S. (2016) Decision Support Systems in Fisheries and Aquaculture: A Systematic Review, ArXiv e-prints [online] https://arxiv.org/abs/1611.08374 (accessed July 2018)

33- Mathisen, B.M., Haro, P., Hanssen, B., Björk, S., Walderhaug, S., 2016. Decision Support Systems in Fisheries and Aquaculture: A Systematic Review. arXiv:1611.08374.

34- Montalvo, I., Izquierdo, J., Pérez-García, R., \& Herrera, M. (2010). Improved performance of PSO with selfadaptive parameters for computing the optimal design of Water Supply Systems. Engineering Applications of Artificial Intelligence, 23(5), 727-735. doi: 10.1016/j.engappai.2010.01.015

35- Parsopoulos KE and Vrahatis MN (2002b) Particle Swarm Optimization method in multiobjective problems. Proceedings ACM Symposium on Applied Computing (SAC 2002), pp. 603-607

36- Parsopoulos, K.E. \& Vrahatis, M.N. (2002a). Particle swarm optimization method for constrained optimization problems. In: Kvasnička, V. et al. (Eds), Proceedings of the second Euro-International Symposium on Computational Intelligence, Košice, Slovakia, pp. 214-220

37- Pelletier, N., \& Tyedmers, P. (2007). Feeding farmed salmon: Is organic better?. Aquaculture, 272(1-4), 399-416. doi: 10.1016/j.aquaculture.2007.06.024

38- Poli, R., Kennedy, J., \& Blackwell, T. (2007). Particle swarm optimization. Swarm Intelligence, 1(1), 33-57. doi: 10.1007/s11721-007-0002-0

39- Rasmussen, R. (2001). Quality of farmed salmonids with emphasis on proximate composition, yield and sensory characteristics. Aquaculture Research, 32(10), 767-786. doi: 10.1046/j.1365-2109.2001.00617

40- Saaty, T.L. (1980). The Analytic Hierarchy Process. McGraw-Hill, New York

41- Seginer, I. (2016). Growth models of gilthead sea bream ( Sparus aurata L.) for aquaculture: A review. Aquacultural Engineering, 70, 15-32. doi: 10.1016/j.aquaeng.2015.12.001

42- Shahidi, F. \& Alasalvar, C. (2010). Marine Oils and Other Marine Nutraceuticals, in: Alasalvar, C., Shahidi, F., Miyashita, K., Wanasundara, U. (Eds.), Handbook of Seafood Quality, Safety and Health Applications. WileyBlackwell, 444-454

43- Shi, Y., \& Eberhart, R. C. (1998). Parameter selection in particle swarm optimization. In Evolutionary Programming VII: Proc. EP98 (pp. 591-600). New York: Springer. doi: 10.1007/BFB0040810

44- Shih, Y. (2017). Integrated GIS and AHP for Marine Aquaculture Site Selection in Penghu Cove in Taiwan. Journal Of Coastal Zone Management, 20(1). doi: 10.4172/2473-3350.1000438

45- Tzeng, G.H. \& Huang, J.J. (2011). Multiple Attribute Decision Making. New York: Chapman and Hall/CRC. https://doi.org/10.1201/b11032

46- Yu, R., \& Leung, P. (2005). Optimal harvesting strategies for a multi-cycle and multi-pond shrimp operation: A practical network model. Mathematics And Computers In Simulation, 68(4), 339-354. doi: 10.1016/j.matcom.2005.01.018

47- Yu, R., \& Leung, P. (2009). Optimal harvest time in continuous aquacultural production: The case of nonhomogeneous production cycles. International Journal Of Production Economics, 117(2), 267-270. doi: 10.1016/j.ijpe.2008.11.001

48- Zander, K., \& Feucht, Y. (2017). Consumers' Willingness to Pay for Sustainable Seafood Made in Europe. Journal Of International Food \& Agribusiness Marketing, 30(3), 251-275. doi: 10.1080/08974438.2017.1413611 